\title{
Administración del Bono Productivo Alimentario por Protagonistas del Municipio de Condega en el año 2015 ${ }^{1}$
}

\author{
Héctor Javier Moncada Barreda ${ }^{2}$ \\ Beverly Estela Castillo Herrera ${ }^{3}$
}

\section{RESUMEN}

El objeto de estudio es el programa productivo alimentario "Hambre Cero" impulsado por el gobierno de Nicaragua a familias protagonistas que habitan en el municipio de Condega y que fueron beneficiadas con el Bono Productivo Alimentario en el año 2015. La investigación es cuantitativa. El objetivo es evaluar qué estrategias implementan las protagonistas durante la administración de los bienes del bono, además conocer los principales factores que inciden en la sostenibilidad de estos recursos, de tal manera proponer una estrategia que permita mejorar el manejo de los recursos: incrementar la producción de alimentos para el autoconsumo, comercializar excedentes de lo producido y fortalecer sus capacidades organizativas lo que permitirá una mayor sostenibilidad y reducir la pobreza en la economía familiar. Se aporta a los programas sociales y a las familias pobres del campo. Los ejes teóricos: pobreza, programa políticas públicas para las mujeres, programas sociales en la reducción de la pobreza y estrategias de administración. Es importante para Nicaragua, aunque ya existen algunos estudios sobre programas sociales y sus aportes en la reducción de la pobreza, sin embargo son pocos los estudios que enfatizan cómo familias administran los recursos de estos programas, principalmente las mujeres.

Palabras claves: Bono Productivo Alimentario, Protagonistas, Administración y Sostenibilidad.

Recibido: 9 de noviembre de 2016

Aceptado: 16 de diciembre de 2016

1 Este artículo se basa en la investigación titulada. Incidencia de las estrategias administrativas implementadas por las protagonistas de los bienes del Programa Productivo Alimentario (PPA) para su sostenibilidad de la economía familiar en el municipio de Condega, durante el año 2015 Tesis para obtener el título de Máster en Gerencia y Administración Pública de UNAN-Managua, FAREM Estelí.

2 UNAN-Managua FAREM-Estelí, Correo electrónico: h_moncada@yahoo.es

3 Doctora en Ciencias Sociales por la Universidad de Zulia, Venezuela. Maestría en Ciencias Sociales por la Universidad de Guadalajara. Docente titular de la UNAN-Managua, FAREM-Estelí. Correo electrónico: beverly.castillo@yahoo. com 


\title{
Application of the Food Production Support Program by Participants in the Municipality of Condega in 2015
}

\begin{abstract}
The target for this research is the Food Production Support Program "Hambre Cero" (Zero Hunger) supported by the government of Nicaragua with participant families living in the municipality of Condega and that were recipients of the Food Production Support Programme in 2015. The investigation is quantitative. The objective of this thesis is to identify the strategies the participants implement as they manage the materials received through the support as well as to identify the main factors that impacted the sustainability of these resources, as a way of proposing a strategy that allows for improved resource management: increase the production of food for home consumption, sale of excess production and strengthen their organizational capacity, which will permit increased sustainability and reduce poverty in the family economy. Contribute to the social programs and the impoverished rural families. The theoretical axis are: poverty, public programming for women, social programming for reducing poverty, and management strategies. This thesis is important for Nicaragua. Although there are already a few studies covering social programs and their contribution to poverty reduction, there are few studies that emphasize how families manage the program resources, especially women.
\end{abstract}

Keywords: Production Bonus, Protagonists, Administration and Sustainability. 


\section{INTRODUCCIÓN}

Los programas de protección social tienden a contribuir a reducir la pobreza en el país y a proteger a la población en situación de vulnerabilidad económica y social ante desastres naturales y choques económicos negativos. Con el cambio de gobierno, como suele pasar en muchos países Latinoamericanos, se dejan de implementar proyectos y diseñan otros nuevos.

En Nicaragua, el Programa Productivo Alimentario (PPA) vino a sustituir al programa Libra por Libra, este último enfocado únicamente en el desarrollo de mercado de la semilla y apoyo a la producción de alimentos básicos. Este Programa esta establecido en el Plan Nacional de Desarrollo Humano (PNDH) impulsado por el Gobierno de Reconciliacion y Unidad Nacional de Nicaragua. En el PNDH ha tenido como alta prioridad el crecimiento económico con aumento del trabajo y reducción de la pobreza y de las desigualdades, la recuperación de valores, la restitución de derechos económicos, sociales, ambientales y culturales del pueblo, sobre todo a los sectores históricamente excluidos y el aumento en las capacidades de las familias nicaragüenses". (GRUN, 2011, pág. 20).

E1 PPA consta de tres componentes: la capitalización a través de la entrega de un Bono Productivo Alimentario (BPA); la capacitación (vía acompañamiento técnico y talleres); y la organización (formación de núcleos y promoción de cooperativas). Una de las principales características del programa es que las beneficiarias directas son mujeres. Se presupone que así hay una mayor repercusión en las familias campesinas y que esto contribuye a la transformación de las relaciones de género en el campo.

Para analizar el desarrollo y los impactos del PPA se aplica los 5 criterios utilizados por la Unión Europea para evaluar sus programas internacionales de desarrollo: Pertinencia, Eficiencia, Eficacia, Impacto y Sostenibilidad. Las recomendaciones del estudio a nivel micro fueron las siguientes, en el ámbito del actual PPA es recomendable involucrar a toda la comunidad local o comarcal en la elaboración final de la lista de familias beneficiadas. De esta manera no solamente se incrementaría el nivel de aceptación del Programa en toda la comunidad, sino también se fortalecerá su capital social, es decir su fuerza para salir de la pobreza en conjunto y en segundo lugar el contenido del Bono debe ser más flexible y de acuerdo a las necesidades reales de las familias pobres. (Kester, 2009, pág. 15)

Las mujeres que acceden al programa se les denominan mujeres protagonistas, por cuanto son ellas las que deciden sobre la administración de sus bienes. Para acceder al programa las mujeres se comprometen a cumplir una serie de requisitos como: tener accesos a una pequeña parcela para mantener los animales, responsabilizarse por los recursos, organizarse, participar de las reuniones y capacitaciones, y ahorrar el $20 \%$ del valor de su bono productivo en un fondo colectivo.

Hay algunas experiencias sobre familias que han sido beneficiadas con el bono, pero no tardan mucho en sostenlo por diferentes razones, pero las más mencionada es que no cuentan con comida para el cuido o que no tienen el espacio para albergarlos. Esto conlleva a que las condiciones de la familia sigan igual, no produce para el autoconsumo mucho menos para la venta. Esto se traduce en un alto grado de insostenibilidad de los recursos, algunas familias terminan perdiendo las gallinas y la cerda porque se les mueren, otras familias presentan animales enfermos a veces por falta de alimentación y/o medicamentos, pero también por mal manejo.

Muchas familias no ahorran el 20\% del total del bono como compromiso adquirido y no se organizan en núcleos tal como lo orienta el programa para fomentar el cooperativismo. Para el aporte del 20\% del fondo revolvente, existe la dificultad que las protagonistas 
destinan parte de los pocos ingresos que poseen a los animales del bono en alimentación y medicamentos y si no hay producción de huevos y lechones en un periodo de tiempo corto no se tendrá de donde extraer el recurso para el debido aporte y seguir manteniendo los animalitos.

La justificación de esta investigación se basa esencialmente en conocer si realmente el Bono Productivo Alimentario ha logrado reducir la pobreza en los hogares de familias rurales del municipio de Condega del departamento de Estelí, Nicaragua, a través de las estrategias que éstas han puesto en práctica durante la administración de los bienes del bono y si ha significado mejorarías en la economía familiar, conocer los logros obtenidos desde su adquisición y hasta donde las mujeres se han logrado organizar según los compromisos adquiridos con el programa. "La pobreza extrema rural supera aproximadamente en 5 veces a la pobreza extrema urbana y la pobreza general rural duplica la pobreza general urbana". (GRUN, 2011, pág. 17) .

En esta investigación se analizan las estrategias implementadas por las familias en la administración del bono, lo que les ha permitido reproducirlos y mejorar sus ingresos a través de la comercialización de los excedentes de la producción y su sostenibilidad. Todo ello, para proponer una estrategia para administrar los bienes del bono que permita mejorar la sostenibilidad a más largo plazo.

\section{Los conceptos teóricos que sustentan el estudio}

El estudio exige conocer sobre el significado de la pobreza desde algunas esferas que pueden ser vistas y que repercuten en diferentes tipos de pobreza en las familias nicaragüense, por eso es que este estudio estará fundamentado en cuatro ejes relacionados a la pobreza, programas sobre políticas públicas para las mujeres, programas sociales en la reducción de la pobreza y estrategias de administración.
Cuadro $N^{\circ}$ 1. Clasificación sobre los conceptos de pobreza

CONCEPCIÓN DEFINICIONES

Pobreza como un Necesidad. La pobreza como concepto material carencia de bienes o servicios materiales, la población "necesita": comida, vestido, combustible o techo.

Un patrón de privaciones. Se define por existencia de patrón de privaciones más que por la privación misma.

Limitación de recursos. La pobreza refiere a circunstancias donde las personas carecen de ingreso, riqueza o recursos para adquirir o consumir las cosas que necesitan.

Pobreza como Nivel de Vida. La idea de "necesidad" situación económica (Bajos ingresos) supone que algunos artículos o bienes son particularmente importantes o necesarios. Esta referido a la experiencia de vivir con menos ingreso o consumo que los demás.

Desigualdad. Las personas pueden ser consideradas pobres porque están en situación de desventaja respecto de otros en la sociedad.

Posición económica. Una "clase" de personas es un grupo identificado en virtud de su posición económica en la sociedad. La clase es un aspecto de desigualdad como característica de la estructura social y no de la desigualdad de recursos o del consumo; los recursos y el consumo son un indicador de posición social.

Pobreza como condiciones sociales.

Clase social. La idea de "clase social" identifica la posición socioeconómica con el estatus socioeconómico. Los roles sociales y ocupacionales constituye la noción de clase.

Dependencia. Algunas veces se considera a la población pobre como aquellos que reciben beneficios sociales debido a su carencia de medios de subsistencia. 
Carencias de seguridad básica. Ha sido definida como necesidad, también puede ser vista como vulnerabilidad ante los riesgos sociales.

Ausencia de titularidades. Carencia de seguridades como una falta de derechos.

Exclusión. Se plantea como un conjunto de relaciones sociales donde las personas están excluidas de participar de una vida social normal.

La pobreza como La pobreza es una privación un juicio moral. juzga que sus condiciones materiales son moralmente inaceptables.

(Spicker, Álvares, \& Gordon, 2009, págs. 291-304).

Todo país que trabaja en función de reducir la pobreza, implementa políticas públicas que se encaminen a superar este fenómeno. En el caso del Gobierno de Reconciliación y Unidad Nacional (GRUN) impulsa la participación plena de las mujeres en todos los procesos de desarrollo político, económico, social y cultural en el país, generados por las Instituciones del Estado y la sociedad en general promueve y visibiliza el liderazgo y protagonismo de las mujeres en todos los ámbitos de la vida. (GRUN, 2011, pág. 90) .

Cuando nos enfrentamos a sectores sociales que sufren pobreza es más difícil lograr que la mujer tenga capacidad y voluntad de participar en diversos espacios ya que no tiene capacidad de decisión. El BPA está orientado a la capitalización de las mujeres campesinas con tenencia de menos 10 manzanas de tierra. PNDH 2012-2016 (GURN, 2011, pág. 221)

Según el Gobierno de Nicaragua (GRUN, 2011, pág. 129) unas de las líneas de acción para la capitalización de las familias, son:

- Ampliar los programas de financiamiento. El GRUN (Gobierno de Reconciliación y Unidad Nacional) continuará expandiendo la capitalización

a través del Bono Productivo Alimentario y el programa Usura Cero a familias del segmento de la población más pobre y vulnerable para mejorar la seguridad alimentaria de las familias e ingresos, contribuyendo a la reducción de la pobreza.

- Entrega en los próximos 5 años de 181 mil nuevos Bonos Productivos Alimentarios (Hambre Cero), tanto para mujeres como para hombres rurales que tienen entre 1 y 10 manzanas de tierra.

Para lograr un buen manejo de los recursos suministrados por los programas sociales es indispensable garantizar una buena administración. En este estudio se entiende la administración como el proceso de trabajar con las personas y con los recursos para cumplir con los objetivos organizacionales. Los buenos administradores llevan a cabo estas funciones de forma eficaz y eficiente. Para Gallardo (2016, pág. 5) ser eficiente significa alcanzar las metas con el menor desperdicio de recursos, es decir, emplear de la mejor forma el dinero, el tiempo, los materiales y a la gente. Además, afirma que ser eficaz significa alcanzar las metas organizacionales.

Según la cartilla elaborada por el Instituto Nacional de Tecnología Agropecuaria (INTA) y e Instituto Nacional Tecnológico (INATEC) (2008, págs. 3-7), el objetivo principal de la producción de aves de corral en las granjas es la obtención máxima de carne y/o huevos por unidad de superficie, al menor costo posible. La crianza de aves de corral debe su mayor desarrollo a la reconocida calidad alimentaría de sus productos (huevo y carne) la que han pasado a ser casi imprescindible en la dieta familiar aún para sectores de limitados recursos.

De igual manera el ganado porcino es importante en el estrato familiar porque constituye una importante fuente de recursos para la economía familiar, tanto como fuente accesible de proteína como para su comercialización. Según (INTA \& INATEC, 2010) 


\section{MATERIALES Y MÉTODOS}

El Universo de Estudio son 250 Bonos entregados a igual número de familias por el Ministerio de Economía Familiar, Comunitaria, Cooperativa y Asociativa (MEFCCA) para el año 2015 en el Municipio de Condega del departamento de Estelí, Nicaragua.

Estos bonos fueron ubicados en cuatro barrios del municipio de Condega que son: Evaristo cruz, Juanita Vizcaya, Linda Vista y el barrio Buena Vista. Además, en veintiocho comunidades que son: Guayucali, Santa Rosa, Darayli, El Hato, Bramadero, Venecia, San Pedro Arenales, San Diego, Santa Lucia, Arenales, San José de Pire, Naranjita, Chaguite Grande, Rodeo de Pire, Potrerillo, Laguna de los Hernández, La Montanita, Las Brumas, San Jerónimo - El Coyol, El Algodonal, El Níspero, Jesús María, Santa Teresa, Jocote Abajo, Jocote Arriba, Santa Rita, Ducuale Grande y San Ramón (MEFCCA, 2015).

La muestra de estudio corresponde a 104 bonos. Esta muestra se toma considerando un $93 \%$ de confianza del universo. Por características propias de la zona se incluye en la muestra 27 comunidades, pero se excluyen la comunidad Santa Rita porque en ese lugar solamente se entregó un bono, con igual criterio se excluye en la zona urbana al barrio Linda Vista.

\section{Etapas de la investigación.}

Investigación Documental: En esta fase se recopiló información para la elaboración del protocolo de investigación: problemas, objetivos, marco teórico y metodología. Se consultaron fuentes en bibliotecas y sitios web relacionados con los temas sobre pobreza, políticas públicas, el Plan Nacional de Desarrollo Humano quinquenio 2012-2016 (PNDH 2012-2016) de Nicaragua e información del Programa Productivo Alimentario "Hambre Cero".
Elaboración de Instrumentos: Los instrumentos de esta investigación se diseñaron considerando los objetivos específicos, la hipótesis y el cuadro de operacionalización de la variable independiente (administración) y la variable dependiente (Sostenibilidad).

Trabajo de campo: Se aplicaron dos instrumentos: entrevista tipo semi-estructurada dirigida a funcionarios del Ministerio de Economía Familiar, Comunitaria, Cooperativa y Asociativa. Las delegaciones del MEFCCA se ubican en las cabeceras departamentales del país como delegaciones, y cuentan con personal en los territorios o municipios de cada departamento. Se entrevistaron a promotores/as, personal técnicos y funcionarios/as encargados del BPA en el municipio de Condega. Se aplicaron las encuestas exclusivamente a las mujeres protagonistas del Bono Productivo Alimentario del municipio de Condega que recibieron el bono en el año 2015, la encuesta además de contener preguntas cerradas, son muy sencillas y de fácil comprensión, para que se facilite la comprensión por parte del encuestado.

Elaboración de informe final: Se elaboró el informe final considerando y analizando los resultados que arrojaron los instrumentos utilizados en la recopilación de la información. El informe final se organiza en base al formato de tesis definida por la UNAN-Managua. En el documento final se incluye una propuesta de estrategia de sostenibilidad dirigida a las familias protagonistas para que mejoren la administración del Bono Productivo Alimentario y alcancen un mayor grado de sostenibilidad.

\section{RESULTADOS Y DISCUSIÓN}

\section{Mecanismos de selección de familias beneficiadas con el BPA}

El programa además de ser dirigido a mujeres, trae consigo criterios definidos para identificar familias que 
son elegibles para optar al bono productivo alimentario y éstos se basan principalmente en tres aspectos; 1. Necesidad, 2. Capacidad y 3. Compromiso. Una vez identificadas las familias como candidatas, El MEFCCA, verifica que las mujeres seleccionadas cumplieran con los criterios establecidos:

- Criterio sobre la necesidad: El programa valora el estado actual de la familia sobre los niveles de pobreza. (Carencia de recursos económicos, materiales, condiciones de la vivienda, otros), si cuenta con cierto capital de trabajo (Aves de corral, cerdos, patos, ganado menos, otros) sean estos para el autoconsumo o para comercializar, también la cantidad de miembros en la familia, Hijos que dependen (Hijos que dependen en edades menores a los cinco años y/o edad escolar), nutrición, empleo, ingresos económicos.

- Criterio sobre Capacidad: Este criterio refiere a capacidades que tiene la mujer rural sobre tenencia de la tierra, cuenta con algunas formas de cultivos de granos básicos, hortalizas, tienen acceso al agua y también si cuenta con apoyo de la familia en actividades propias del hogar (cuido y manejo de los animales, cultivos de granos básicos u hortalizas).

- Tiene un área de terreno apta para el cuido y manejo de los animales (gallinas, cerdos o vaca).

- Cultivos: La familia siembra frijoles, maíz, maicillo, hortalizas, u otros productos, como principal fuente de alimentación.

- Acceso al agua: La familia cuenta con agua de pozo, rio, quebrada, servicio público, u otra forma de garantizar el vital líquido para el consumo humano y de los animales.

- Apoyo de la familia; valorar si la mujer la protagonista que administra el bono, cuenta con ayuda de la familia, para el cuido y manejo de los animales.
- Criterio sobre el compromiso: son varios los compromisos adquiridos por la protagonista del bono: organizarse en núcleos asociativos de la comunidad con otras protagonistas, asistir y participar en las capacitaciones impartidas por el MEFCCA antes de recibir el bono, poner en práctica conocimientos según su experiencia en producción agrícola y los conocimientos que se acrecentaron sobre el cuido y manejo de aves de corral y cerdos a través de las capacitaciones recibidas, ahorrar el 20\% del monto total del bono como fondo revolvente, asistir y participar en los núcleos asociativos comunitarios, reproducción de las gallinas y cerdos, producción de huevos, administrar de manera eficiente para garantizar el autoconsumo y comercializar los excedentes de la producción.

\section{Principales factores que inciden en la administración y sostenibilidad del BPA}

Experiencia productiva: Todas las mujeres encuestadas afirman tener más de un año de manejar animales domésticos como gallinas y cerdos. El estudio revela que hay experiencia productiva por parte de las protagonistas del bono, sin embargo, también las encuestas revelan que la principal causa de muerte de las gallinas y las cerdas es por enfermedad, y otras mujeres afirman que es por falta de comida y calidad de los animales.

En el estudio se demuestra que son pocas mujeres que compraron comida después que se agotó el concentrado del bono, demostrando que no tenían otras formas o alternativas de solución, es decir muchas familias cosechan maíz y maicillo que requieren, tanto para el consumo familiar como para los animales. Pocas mujeres optaron por elaborar concentrados caseros.

Mano de obra: Al seleccionar a las familias, el MEFCCA identifica sus necedades, verifica si tienen capacidad enfocada en el apoyo que pueden recibir 
de hijas e hijos mayores de catorce años, conyugue $u$ otros familiares.

Las familias que afirman que la ayuda la consideran significativa porque las mujeres por sí solas se les dificulta el cuido y manejo de los animales porque tienen muchos quehaceres en el hogar, pero cuando la familia ayuda se administran mejor los bienes y tienen mejores resultados.

Mientras que las familias que les es indiferente el apoyo, se debe a que se sienten con suficiente capacidad de cuidar y manejar los animales y también hacer otras tareas en el hogar de manera solitaria. Pero ronda la duda que requiere apoyo, quizás no ha logrado el suficiente liderazgo en el hogar como jefa de familia para motivar al resto de la familia para que se incorpore a las actividades del hogar.

Las mujeres que contestaron que los demás miembros de la familia no ayudan, enfatizaron que no hay interés en el cuido de los animales del bono. Consideramos similar al análisis anterior, quizás sea la falta de liderazgo de la mujer como jefa de familia que incida en la falta de apoyo. Estas familias en estudio tienen la capacidad en recursos humanos para una mejor administración, criterio indispensable al ser seleccionadas para optar al BPA.

Alojamiento: Uno de los principales criterios del programa es que las protagonistas del BPA tengan acceso a una parcela de tierra o patio donde cuidar los animalitos (aves y cerdos). Se puede afirmar que las protagonistas si cuentan con suficiente espacio para el cuido y manejo de los bienes del bono, ya que no se requiere de mucho espacio, perfectamente pueden reproducirlos (cerdos y gallinas) y también pueden comercializar.

Infraestructura (Gallineros y Chiqueros): La falta de gallinero y chiqueros ha provocado en gran medida la poca sostenibilidad del bono. El estudio demuestra que las protagonistas del bono no todas cuentan con gallineros o chiqueros para alojar los animales y que aquellas que tienen se encuentra en mal estado.

Alimentos y agua: En las comunidades de incidencia del bono el estudio revela que las familias cuentan con agua para el consumo humano, y una parte la destinan a los animales. En cuanto a la comida; el programa incluye tres quintales de concentrado, y dos de ellos son para el cuido de la cerda, sin embargo, ésta cantidad solamente alcanza para mantener a los animales los primeros sesenta días aproximadamente, después las protagonistas tendrán que alimentarlas con sus propios recursos.

Cuando se abordó este tema con las familias protagonistas, la mayoría afirma que los animales vienen a competir con la comida de las familias y que a veces no hay la posibilidad de alimentar a los animales. Identifican las causas como: tiempo de escasez de maíz y maicillo en la zona en veces por falta o exceso de lluvia.

Calidad (raza mejorada): Aunque el estudio revela que del total de mujeres encuestadas el 21\% dicen que la principal causa de muerte de los animales del bono es por la raza y que no se adaptan a la zona, prefieren mejor animales criollos, sin embargo el Instituto Nacional de Tecnología Agropecuaria (INTA) sostiene que las gallinas y las cerdas de raza mejorada que entrega el MEFCCA se adaptan a cualquier zona o clima siempre y cuando se les trate adecuadamente.

Fondo revolvente: De las 104 familias encuestadas, el 40\% (42 mujeres) ahorraron según compromisos adquiridos con el programa. Las 64 familias restantes dicen que no ahorraron por diferentes causas: falta de organización en la comunidad, falta de exigencias del MEFCCA, para asumir gastos en alimentación y medicamentos de los animales. 
Registros y controles: Se puede afirmar que la falta de registros ocasiona que las administradoras del BPA no logren percibir si están o no invirtiendo recursos para el cuido y manejo de los animales, no pueden determinar con seguridad si están recibiendo ingresos significativos a través de comercialización de huevos y lechones.

\section{Reproducción y comercialización}

Reproducción y existencia de gallinas: El estudio se llevó a cabo casi un año después de que las protagonistas recibieran el bono. Se encuestaron 104 de un total de 280 protagonistas quienes en la actualidad sólo cuentan con 388 gallinas, sin incluir gallos. No hubo multiplicación de estos bienes, más bien hubo una disminución de aproximadamente 548 gallinas en relación a lo esperado. Es decir, que de las 104 protagonistas encuestadas todas recibieron 9 gallinas y un gallo, lo que suman 936 gallinas y 104 gallos. A escasos doce meses deberían tener al menos la misma cantidad o más de la recibida para demostrar la multiplicación de los bienes.

Producción y autoconsumo de huevos: Las gallinas procedentes del bono no todas están produciendo huevos, sin embargo los resultados de la encuesta revela que existe una producción de 68 docenas de huevos por familia. La encuesta arroja que 55 docenas de huevos son destinadas al consumo, es decir que las familias consumen casi el $81 \%$ de la producción de huevos. Lo cual representa un ahorro en dinero y la mejora de la dieta alimenticia.

Existencia de cerdos: Según las protagonistas encuestadas, en la actualidad sólo existen 72 cerdas y 3 cerdos del bono, en este caso es diferente al bono de las gallinas, porque aquí lo que se pretende demostrar es que si las familias aún tienen interés en seguir trabajando con este recurso (cerdos), pero a simple vista se nota que disminuye la cantidad de cerdos en existencia por familia en relación a lo esperado. Es decir, cada protagonista recibió una, lo que suman 104 cerdas. A escaso doce meses después de haber recibido el bono, debería cada familia tener una cerda o más de una.

Sin embargo, la cantidad esperada de lechones producidos asciende a 1,560 (un mil quinientos sesenta lechones) es decir de 104 cerdas con dos parto al año y un promedio de 15 lechones por camada, con un margen de error del 15\% como imprevistos (muerte de la cerda, muerte de las crías, sólo un parto en el año, camadas con índices mucho menores del promedio establecido, otras causas), significa dejar de producir 234 lechones.

Se puede concluir que las protagonistas del bono dejaron de reproducir más o menos 234 lechones siendo una de las causas principales el bajo rendimiento por camada que puntea un promedio de 10 lechoncitos por cada parto.

\section{Comercialización}

Venta de huevos: Las 104 familias encuestadas dicen vender al menos 13 docenas de huevos a la semana, significando que de la producción de huevos semanal 68 docenas sólo el 19\% lo destinan a la comercialización.

Venta de lechones: De las 104 protagonistas encuestadas 80 de ellas reprodujeron cerdos, sumando un total de 861 lechones, la información suministrada por estas ochenta protagonistas sobre la cantidad de lechones vendidos suma un total de 660 seiscientos sesenta cerdos. Nos detendremos un poco para responder a una interrogante ¿por qué esta cantidad y no la producida? Según las protagonistas del bono la cantidad de cerdos producidos no es igual a los vendidos porque un lechón lo comprometen con el dueño del semental y otro lecncito lo dejan para la crianza, quizás otro lo regalen, pero también visualizan la posible muerte de un lechoncito durante el desarrollo es decir que de cada parto se dejan de dos a tres lechones sin importar la cantidad por camada. 


\section{Organización}

Se comprobó que una buena parte de las protagonistas encuestadas desconocen el surgimiento de cooperativas a través mujeres organizadas. Aunque, según funcionarios del Ministerio de Economía Familiar se logró constituir en el año 2011 una cooperativa proveniente de mujeres organizadas en núcleos, y que en la actualidad están ejecutando un plan de inversión, que su principal fuente de financiamiento es el MEFCCA a través del programa Integral de Emprendimientos en Centro América y Republica Dominicana (CENPROMYPE)

Este funcionario del Ministerio manifiesta que en el año 2008, 44 mujeres provenientes de 04 comunidades del Municipio de Condega (Ducuale, El Mojón, San Diego y La Montañita), se conformaron en un núcleo cuyo nombre fue Unidas Lucharemos, para participar en el programa del Gobierno de Reconciliación y Unidad Nacional con BPA (Bono productivo Alimentario), por medio del cual fueron capitalizadas con la entrega de bonos que incluían cerdos, vacas y aves. Todo con el objetivo de combatir la pobreza, recuperación de los saberes, la autoestima y la soberanía alimentaria.

Posteriormente a finales del año 2011, el núcleo decide conformarse en una organización legalmente constituida, la cual se denomina Cooperativa Multisectorial Unidas Lucharemos Mejor, R.L. (COMULUM, R.L), se logra conformar con un total de 23 socias y obtuvieron su personería jurídica el 06 de diciembre del año 2011.

Actualmente la cooperativa cuenta con una tienda campesina, ubicada en la comunidad de San Diego a $5 \mathrm{~km}$ al Este de Condega sobre la carretera Yalí, este municipio pertenece al departamento de Estelí, donde ofertan una variedad de productos (Abarrotería, insumos agrícolas, entre otros). También se dedica a la comercialización de carne de cerdo y sub productos del mismo, los cuales son comercializados en la comunidad.
Participación de las protagonistas: Uno de los criterios de selección de las mujeres para ser merecedoras del bono es que deben comprometerse a organizarse en núcleos y también participar en las reuniones, pero el estudio revela que sólo un $64 \%$ de las mujeres encuestadas participan en reuniones desde los núcleos asociativos comunitarios.

Asistencia técnica: De las 104 protagonistas encuestadas 47 dicen no haber recibido asistencia técnica por parte de los técnicos del Ministerio de Economía Familiar, mientras que las 57 familias restantes que han logrado recibir afirman no estar tan satisfechas. Sin embargo, cuando se abordó este tema con funcionarios del MEFCCA explican que en el municipio solo hay tres técnicos y que es imposible garantizar asistencia técnica a cada una de las protagonistas.

Según los técnicos la estrategia es capacitar a promotoras que habitan en las comunidades de las protagonistas sobre cómo cuidar y manejar los animales del bono, asignan a cada una, cierta cantidad de núcleos a quienes visitaran de manera permanente para responder a lo inmediato aquellos casos que puedan resolver en el lugar y los casos más complejos los remiten a la delegación a través de gestiones pertinentes.

\section{Propuesta (Granja Comunitaria)}

En este documento se tratará la "Sostenibilidad del Bono Productivo Alimentario", por lo cual podríamos pensar que lo que se desea es que sea sostenible, pero no perder de vista que lo que pretende no es mantenerlo, sino los beneficios que este proporciona a través de una mejor explotación de los bienes entregados.

El Bono Productivo Alimentario, está dirigido a las mujeres jefas de familias con apoyo de la mano de obra familiar. Cabe mencionar que estas familias tienen capitales que aún no están siendo explotados en su totalidad; como parte de los capitales podemos 
mencionar el capital humano, el capital natural y en cierta medida el capital social.

Objetivos de Sostenibilidad: Incrementar los bienes adquiridos en el BPA a través de iniciativas de granjas comunitarias.

Objetivos Operativos: Fomentar en las familias iniciativas de negocio que incrementen los bienes recibidos por el BPA a través de planes de negocio.

\section{Principales temas a trabajar:}

\section{Organización}

- Promotoría Social.

- Organización en Núcleos Asociativos.

- Cumplimiento del Ahorro.

- Participación Activa en talleres de Capacitación y/o Reuniones.

- Espacios de Intercambios de Experiencias exitosas en Ferias, Mercados Campesinos Locales y Administración Eficiente del BPA.

- Rescate de Cultura Nicaragüense sobre la presentación del producto para la venta; ejemplo venta de huevos en tuza, cuajada en hojas de guarumo.

\section{Producción}

- Visión Empresarial (Masificar los bienes físicos entregados por el BPA).

- Manejo y cuido eficiente de los animales (Infraestructura).

- Alimentación versus Producción (Nutrición).

- Rentabilidad.

- Establecimiento de parcelas para el cultivo de alimentos Alternativos: (Ejemplo Frijol gandul, caballero, terciopelo, etcétera).

- Buenas Prácticas de Manufactura.

\section{Comercialización}

- Valor Agregado a los bienes del BPA para la comercialización.
- Empaque para la presentación de productos.

- Mercado Local.

- Participación en Ferias.

- Diversificación de Productos.

- Intercambios de Experiencia.

- Rescate de Cultura Nicaragüense sobre la presentación del producto para la venta; ejemplo venta de huevos en tuza ${ }^{1}$, cuajada en hojas de guarumo ${ }^{2}$.

- Plan de Negocio.

\section{Lineamientos de trabajo Generales:}

- Identificar el liderazgo de las mujeres para la conformación de las promotoras del BPA.

- Realizar plan de capacitación permanente a promotoras del BPA que contribuya al empoderamiento como promotoras.

- Realizar campañas de visitas a las familias beneficiarias del BPA para concientizarlas sobre el ahorro del $20 \%$ y a la vez motivarlas para que se haga efectivo el ahorro.

- Apoyar iniciativas locales a través de planes de negocio.

- Incentivar la inversión a través de la implementación de granjas avícolas y porcinas en pequeños grupos de mujeres para la comercialización de carne y huevos.

- Promoción de la producción diversificada y mejoramiento de la calidad de productos en la finca.

- Fortalecimiento de la gestión de inteligencia de mercados dando valor agregado a los bienes del BPA.

- Realizar convenios de comercialización con las municipalidades para la utilización de infraestructuras públicas como los parques de ferias

1 Tuza; es la hoja que envuelve la mazorca de maíz. Las familias campesinas la usan para diferentes actividades, una de ellas envolver huevos de gallinas para protegerlos.

2 Hoja del árbol de guarumo, las familias campesinas usan esta hoja para envolver la cuajada dando un aroma y sabor muy característico. 
para el establecimiento de tiangues permanentes de exhibición y promoción de sus productos.

- Promoción de valores de una comercialización responsable, que contribuyan a que seamos parte del proceso.

- Implementar iniciativas de centros de producción comunitarias de concentrados caseros para animales.

- Sistematizar experiencias exitosas de mujeres que han administrado de forma eficiente el BPA.

\section{Instrumentos de control y seguimiento.}

Para establecer control exacto de los lineamientos a realizar; desde los núcleos comunitarios se deben de mantener registros permanentes de información, dicha información deberá ser monitoreada por las promotoras del BPA.

Para el monitoreo se debe de establecer formatos estándares, dichos formatos tendrán como objetivo primordial la recolección y registro de la información que evidencie que se están dando cumplimiento a los lineamientos generales de la estrategia de Sostenibilidad del BPA. Se debe de registrar la información de acuerdo al plan de Acción que se establezca para el buen funcionamiento del BPA.

Ejemplos de instrumentos para el control y seguimiento citamos los siguientes:

- Plan de Ferias Locales y Nacionales.

- Participación en Ferias.

- Actas de Reuniones y/o Asambleas.

- Registro de gastos para la alimentación y medicina de los animales.

- Registro de ingresos provenientes de la venta de los excedentes de la producción.

- Manejo del Ahorro.

- Manejo de los intereses y mantenimiento de valor del ahorro.

- Convenios y/o Alianzas.

\section{CONCLUSIONES}

El Gobierno de Reconciliación y Unidad Nacional de Nicaragua define los criterios para seleccionar familias e incluirlas en el Programa Productivo Alimentario Hambre cero a través del principal instrumento de capitalización que es el Bono Productivo Alimentario. Las familias seleccionadas deben tener necesidad, capacidad y adquirir algunos compromisos con el programa.

Independientemente que el gobierno seleccione a las familias determinando las capacidades de éstas en manejar y cuidar los bienes del bono, el estudio revela la existencia de muchos factores que inciden en la administración y sostenibilidad, siendo éstos los principales:

- Experiencia productiva (cuido y manejo de los animales).

- Disponibilidad de mano de obra familiar,

- Tamaño de la propiedad,

- Infraestructura (gallineros y chiqueros),

- Capacidad de alimentación, agua y medicamentos).

- Ahorro del 20\% como capital de trabajo.

- Calidad de los animales adquiridos (raza mejorada).

- Registros de ingresos y egresos.

También inciden otros factores, pero en menor grado o indirectamente, como capacitaciones que han recibido las protagonistas y la asistencia técnica, el hecho de ser mujer la administradora del bono, la edad de las protagonistas, migración de protagonistas a otras comunidades o al otro lado de las fronteras nicaragüense, entre otras.

En cuanto a las capacidades organizativas se logra demostrar que aún falta mucho para cumplir con este componente en ambas vías: programa como gobierno y protagonistas del bono. Se muestra que hay cierto grado de deserción de algunas protagonistas que no participan en reuniones desde los núcleos asociativos 
comunitarios, que se debe mejorar el ejercicio del liderazgo, no solo por parte de los líderes y lideresas de la comunidad, sino, también por parte Ministerio encargado del programa y la red de promotoría social.

Poca cohesión por parte de los núcleos para que sus integrantes decidan subir al siguiente peldaño ya sea para organizar su propia cooperativa u otra iniciativa de negocio. Solamente ha surgido una cooperativa proveniente de núcleos asociativos en Condega y tomando como referente que el programa está desde el año 2007.

Las protagonistas dicen haber acrecentado sus conocimientos con las capacitaciones que recibieron antes que el gobierno les entregara el bono, sin embargo, dicen sentirse un poco abandonadas por el MEFCCA porque casi nunca las visitan para darles asistencia técnica y cuando logran visitarlas la asistencia no es muy buena.

Pasado un año de administración del bono, las protagonistas no logran producir lo esperado, presentan bajos rendimientos en la producción de huevos y lechones. Pocas mujeres compraron gallinas para reponer aquellas que se perdieron, dato que puede interpretarse que hubo poco interés en intensificar el rubro. Pero no todo es malo, porque que buena parte de la producción de huevos las familias la destinan para el autoconsumo.

La comercialización de huevos y lechones se hace en la misma comunidad, no trasciende a otros mercados, la venta de huevos es significativamente baja en relación a lo esperado, pero contrario la venta de lechones presenta un mejor comportamiento.

En vista que la hipótesis señala que la sostenibilidad del bono obedece a la forma de adminístralo por parte las mujeres protagonistas, esto permite decir que las estrategias que hasta ahora han puesto en práctica las administradoras del bono no necesariamente determinan la sostenibilidad de los bienes, hay muchos factores que intervienen, hasta la variabilidad de las zonas, por ello es que se cumple parcialmente la hipótesis.

Sin duda alguna, aunque el estudio revela que el bono es poco sostenible y que no ha generado grandes ganancias económicas, pero no se puede obviar que implica la participación real y efectiva de las mujeres como actoras directas y conlleva a una transformación de relaciones y estilos de vida con el fomento y la promoción de nuevos valores que reivindiquen los derechos de todas las mujeres y principalmente las jefas de familias.

Se propone una estrategia de sostenibilidad para que las familias protagonistas del Bono Productivo Alimentario implementen otras formas de administración y poner en práctica acciones que lleven a una mayor sostenibilidad de los bienes.

\section{BIBLIOGRAFÍA}

Gallardo, E. (24 de Marzo de 2016). Fundamentos de Aministración de Empresas. Obtenido de www.sld. $\mathrm{cu} /$ galerias/doc/sitios/infodir/administracion_de empresas.doc.

GRUN. (2011). Plan Nacional de Desarrollo Humano 2012-2016. Nicaragua .

GRUN. (2016). Plan Nacional de Desarrollo Humano. Nicaragua.

INATEC, I. (10 de Agosto de 2008). Manejo Eficiente de las gallinas de patio. Obtenido de http://www. inta.gob.ni/biblioteca/images/pdf/manuales catalogos/MANEJO\%20EFICIENTE\%20DE\%20 GALLINAS\%20DE\%20PATIO.pdf

INATEC, I. (11 de Agosto de 2008). Manejo Eficiente de los Cerdos. Obtenido de http://www.fao.org/3/ a-as542s.pdf: http://www.fao.org/3/a-as542s.pdf

MEFCCA. (2015). BPA. Bono Productivo Alimentario, Condega.

Paul, K. (10 de Abril de 2016). Informe Evaluativo (2007- 2008) PPA. Obtenido de nicaragua. 
nlembajada.org/.../estudio-evaluativo-hambrecero-ppa-2007.

Spicker, P., Álvares, L., \& Gordon, S. (2009).
Definiciones sobre Pobreza; doce grupos de significados. Obtenido de www.bibliotecavirtual. clacso.org.ar/libros/clacso/crop/.../065pick.pdf 